\title{
Design and Analysis of a Dynamic Code Division Multiple Access Communication System Based on Tunable Optical Filter
}

\author{
Mohammad M. N. Hamarsheh, Hossam M. H. Shalaby, Senior Member, IEEE, and \\ Mohamad Khazani Abdullah, Member, IEEE
}

\begin{abstract}
A dynamic optical code division multiple access (DOCDMA) communication system is proposed for high-bandwidth communication systems. An implementation of the system is proposed based on a fast tunable optical filter (TOF) in each encoder and decoder. This technique actively modulates the central wavelength of a TOF according to a functional code at the transmitter during the bit period before the transmission of the data. The system is modeled and analyzed taking into account multiple access interference (MAI), thermal noise, and phase-induced intensity noise (PIIN). The performance of this system is compared to that of a spectral amplitude coding system that uses either a Hadamard code or a modified quadratic congruence (MQC) code. The results show that the proposed DOCDMA system reduces the PIIN effect on the performance of the system and improves the bit error rate (BER) performance at a large number of users. Furthermore, it is found that when the effective power is large enough, the MAI becomes the main factor that limits system performance, whereas when the effective power is relatively low, both thermal noise and PIIN become the main limiting factors with thermal noise having the main influence.
\end{abstract}

Index Terms-Multiple access interference, optical code division multiple access (CDMA), optical fiber communication systems, spectral amplitude coding.

\section{INTRODUCTION}

$\mathbf{O}$ PTICAL code division multiple access (CDMA) systems are one class of systems that provide solutions to multiple access in all-optical communication networks. In addition to the good performance at high number of users and asynchronous access to the network, optical CDMA systems provide the users with high security by coding the data before transmission and at the same time using this coding to recover the data at the receiver [1], [2].

Many optical CDMA communication schemes have been proposed in the last two decades. Early optical CDMA systems

Manuscript received October 1, 2004; revised August 5, 2005. This work was supported in part by the Malaysian Ministry of Science, Technology, and Innovation under IRPA Grant 02-02-04-T001.

M. M. N. Hamarsheh is with the Department of Computer and Communication Systems Engineering, University Putra Malaysia, Serdang, Selangor D. E. 43400, Malaysia, and also with Photronix (M) Sdn Bhd, Cyberjaya, Selangor D. E. 43400, Malaysia (e-mail: hamarsheh@photronixm.com).

H. M. H. Shalaby is with the Department of Electrical Engineering, University of Alexandria, Alexandria 21544, Egypt (e-mail: shalaby@ieee.org).

M. K. Abdullah is with the Department of Computer and Communication Systems Engineering, University Putra Malaysia, Serdang, Selangor D. E. 43400, Malaysia (e-mail: khazani@eng.upm.edu.my).

Digital Object Identifier 10.1109/JLT.2005.858232 coded the incoherent pulses in time domain and recovered the data at the receiver using taped delay lines [3]-[5]. The performance of these systems is poor because of the correlation properties of the special unipolar codes used [6]. These systems also require optical summation that causes considerable losses [7]. Ladder networks are introduced to reduce the splitting loss problem [8]-[11], but the encoder/decoder design limits the number of different codes that could be generated. Coherent systems are also proposed; they allow the use of bipolar codes that can be designed in such a way that some or all of the multiple access interference (MAI) can be cancelled [12]-[17]. However, incoherent systems are more attractive for their simplicity.

Another incoherent technique for optical CDMA is based on spectral amplitude coding (SAC) systems [18]-[21]. In these systems, the spectrum of the broadband sources is encoded. In order to achieve passive optical CDMA with a much better performance, MAI can be canceled by using code sequences with fixed in-phase cross correlation. The system, however, still have the phase-induced intensity noise (PIIN) as the main parameter that limits its performance. Some codes have been proposed to suppress the effect of the PIIN, but it is still the main source of noise [22]-[28].

Recently, frequency hopping systems have been proposed [29]-[33]. It utilizes both time and frequency domains for encoding the optical signal, thus allowing for more flexibility in generating the codes and better reduction of MAI and PIIN. However, they all suffer from complicated configurations due to the use of many elements in each of the encoder and decoder.

In this paper, we propose a dynamic optical CDMA (DOCDMA) system employing high-speed tunable optical filters (TOFs) [34]-[40]. The encoder modulates the central frequency of the pulse optical signal according to a functional code. The synchronized system can recover the encoded data by a matched TOF at the receiver. Only one TOF is required in each of the encoder and decoder. Furthermore, DOCDMA signals interfere only during the time of intersection between the functional codes driving the TOFs. This system utilizes an active correlation receiver where the integration time after the photodetector is in $T$ seconds. Thus, this receiver requires a lower speed electronic design compared with passive correlation receivers [41].

Functional codes are chosen to minimize the intersections; thus, the time limitation decreases both the PIIN and MAI effect on the bit error rate (BER) performance. 


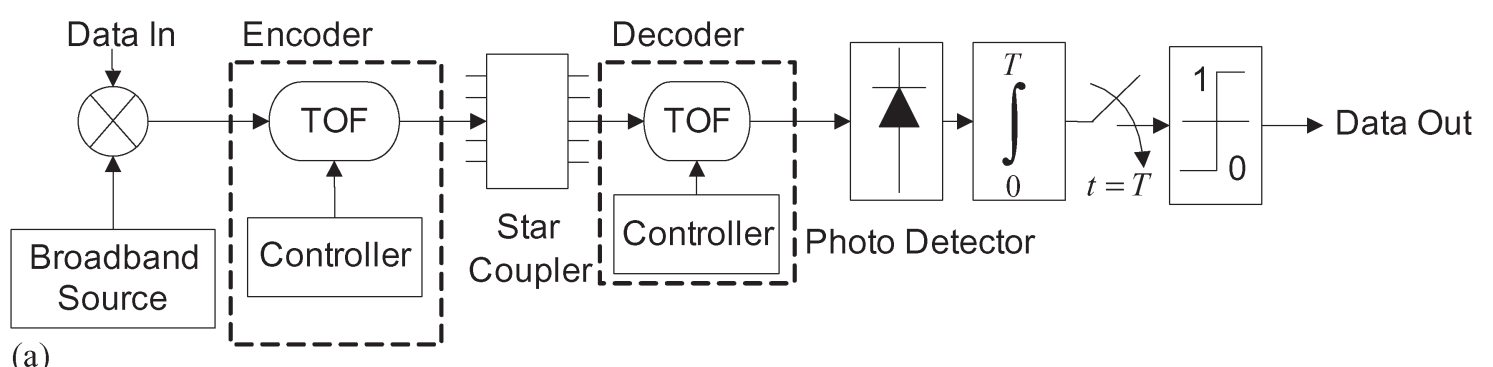

(a)
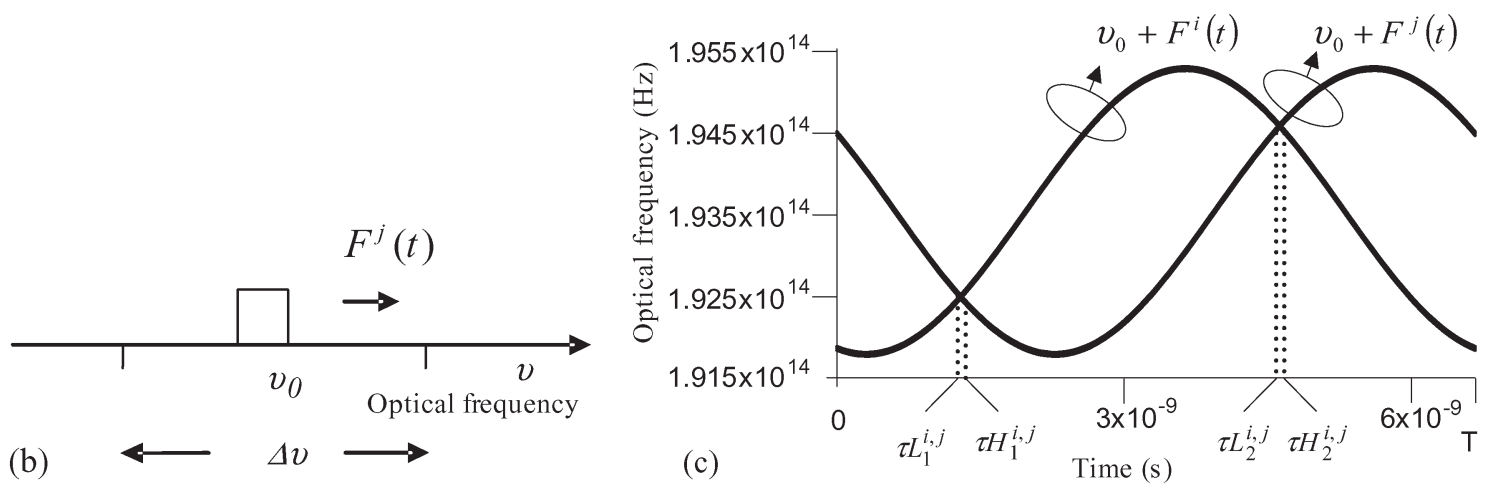

Fig. 1. (a) Transmitter and receiver block diagram of a DOCDMA system. (b) Optical spectrum of a signal from one of the users. (c) PSD for two users as a function of time and frequency.

It has been found that the intensity effect is effectively suppressed using this system and the main noise source for this system is the MAI. However, the system performance is still better compared to the SAC systems recently proposed [25], [26].

The scheme uses codes based on wavelength modulation implemented with a single fast TOF in each encoder and decoder. This allows for easy reconfiguration of the encoder and decoder to any codes without the need for any hardware modification.

The rest of this paper is organized as follows. Section II is devoted to system configuration and description. In Section III, we describe the system mathematical model. In Section IV, we analyze and discuss the DOCDMA system performance, taking into consideration the interference effect, thermal noise, and PIIN. The system numerical results are also compared with those of former systems in Section V. Finally, conclusions are drawn in Section VI.

\section{System CONFIgURATION AND DeSCRIPTION}

The block diagram in Fig. 1(a) shows the DOCDMA configuration. The broadband signal from the light source is ON-OFF keying (OOK) modulated with the binary data. The transmitter sends a pulse with spectral distribution varying with time if the data bit value is "1"; otherwise, no power is transmitted. For each data bit of " 1 ," encoder $j, j \in\{1,2, \ldots, K\}$, where $K$ is the number of simultaneous users, will filter the spectrum of the pulse at a central wavelength that varies according to a functional code $F^{j}(t)$. The encoder is a fast TOF controlled with an electrical signal that represents the functional code. Signals transmitted from all synchronized users will be mixed up in the network before received by all users. At the receiver, the composite signal is decoded by a matched TOF. Then, the signal passes through a photodetector, an integrator, and a threshold decision to recover the data transmitted.

The source spectra are assumed to be flat over the bandwidth of $v_{0} \pm \Delta v / 2$, where $v_{0}$ is the central optical frequency and $\Delta v$ is the system bandwidth. Ideal masking at the TOF is also assumed, and each user is considered to have the same effective average power at each receiver.

Fig. 1(b) shows the spectrum of the $j$ th user's transmitted signal when the data bit is "1." The spectrum is similar to that of an ideal filter with central frequency varying with time according to a functional code. The proposed functional code family $F(t)$ is a sine function family with the same frequency and different phase shifts. Fig. 1(c) shows an example of the spectrum for two users at the input of the decoder during one bit period when both users are sending a bit of "1." The TOFs of the decoders are synchronized in time with a phase shift related to the functional code for each one of them. The output of the decoder is therefore the signal that has the same phase shift with some interference noise at the points of intersection with other users.

\section{Code Construction}

The main criterion in functional code construction is to minimize as much as possible the number of intersecting points between any pair of functions since they increase the interfering power between users. The area of intersection between any two functions, which is related directly to the value of interfering power, is also an important parameter in the construction of the functional codes. In our proposal, we suggest the use of shifted sine functions (SSFs) to alter the optical central frequency $\left(v_{0}\right)$ for coding. The SSF is given by

$$
F^{j}(t)=\frac{\Delta v}{2} \sin (2 \pi f t-j \varphi), \quad j \in\{1,2, \ldots, K\}
$$


where $f$ is the frequency of the functional code, and $\varphi$ is the phase shift between different functions. SSFs are proposed for their simplicity and the possibility of achieving the large number of required codes by reducing the phase shift.

The TOF in DOCDMA should be able to follow the functional code driving the filter. The required speed of the TOF and its controller is defined as the derivative of the code and is given by

$$
S^{j}(t)=\Delta v \pi f \sin (2 \pi f t-j \varphi) .
$$

It is directly proportional to the frequency and amplitude of the functional code.

The functional codes proposed start and stop at the same central wavelength during the data bit interval $(T)$ for smooth modulation of the TOF and its controller. This limits the frequency of the code to be an integer value of $(1 / T)$. For these reasons, we use the smallest frequency possible for the SSF that equals the data bit rate. The phase shift between codes $(\varphi)$ is related to the TOF bandwidth required and the code size. A smaller phase shift results in a larger family of codes, but it requires a smaller bandwidth for the TOF. The phase shift of SSF functions is chosen to be $2 \pi / 169$, which results in 169 different codes that are the same as the cardinality of modified quadratic congruence (MQC) family of codes with $p=13$ [23], for comparison purposes (with MQC).

Using this code with a $50 \mathrm{Mb} / \mathrm{s}$ DOCDMA communication system that uses a light source with 30-nm bandwidth, the filter is required to scan the wavelength range with a maximum speed of $4.7 \mathrm{~nm} / \mathrm{ns}$. The small data bit interval of the high data bit rate system requires fast TOF or functional code with smaller tuning range suitable with the speed of the TOF. Nevertheless, TOFs that can scan tens of nanometers within a few nanoseconds have been reported [34], [40].

Fig. 2(a) shows an example on the power spectral density (PSD) for a signal at the output of one decoder assuming ten users are sending a data bit of " 1 " at the same time. The power is doubled at the points of intersection between the decoder functional code and the other nine codes. These intersections represent the interference between users and will cause shots in the value of the photocurrent at the output of the photodetector as shown in Fig. 2(b). The areas of intersection between the users increase the power coupled between those users, which represent the in-phase cross correlation.

\section{DOCDMA SYSTEM MODEL}

Like typical optical CDMA communication networks, we consider that $K$ simultaneous active users share the same optical fiber network. Each data bit is encoded onto a functional code $F^{m}(t)$ at the encoder of user $m$. The PSD $G(v, t)$ of the signal at the receivers' input is the sum of all active users' transmitted signals

$$
G_{m}^{i}(v, t)=\frac{P_{r}}{\Delta v} \sum_{j=1}^{K} b^{j} \operatorname{rect}\left(\frac{v-v_{0}-F^{j}(t)}{\mathrm{BW}}\right)
$$

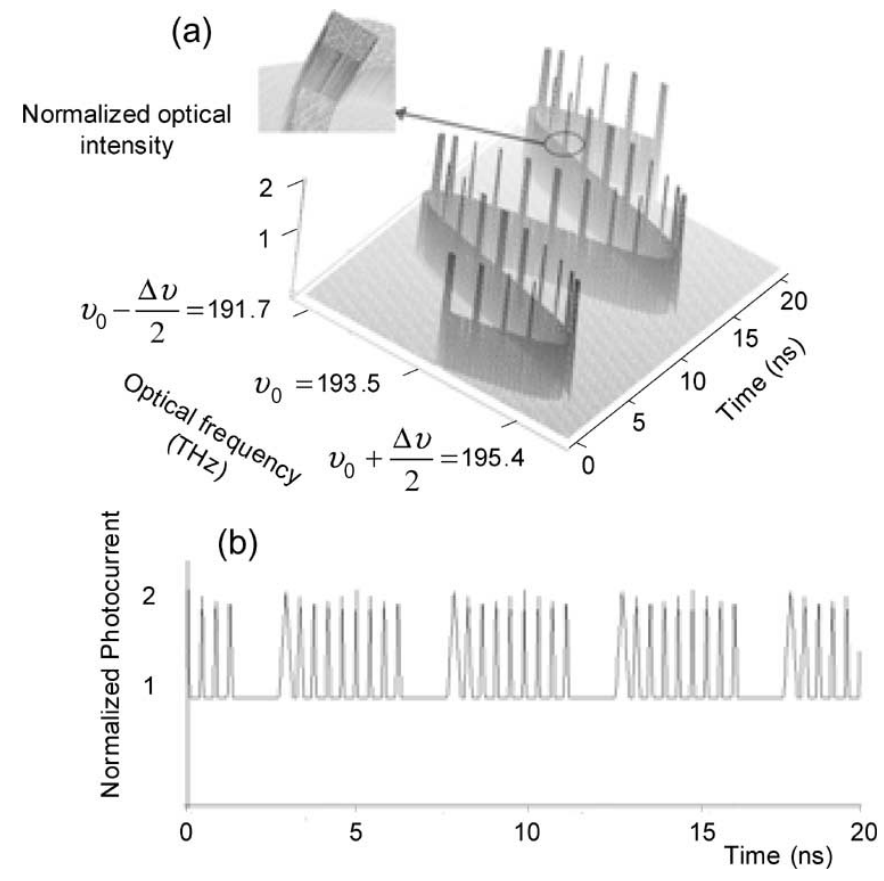

Fig. 2. (a) PSD of one signal at the output of one decoder using the proposed SSF codes. (b) Photocurrent at the output of the photodetector of the same receiver.

where $P_{r}$ is the effective power of a broadband source at the receiver including the losses of transmission and star coupler, $\operatorname{rect}\left(\left(v-v_{0}\right) / \mathrm{BW}\right)=u\left(v-v_{0}+(\mathrm{BW} / 2)\right)-u(v-$ $\left.v_{0}-(\mathrm{BW} / 2)\right), u(v)$ is the unit step function, $\mathrm{BW}$ is the bandwidth of the TOFs, and $b^{j}$ is the data bit value of user $j$.

The receiver applies a synchronized matched filter in decoding the incoming signal to extract the desired user's bit stream. The decoder output and the photocurrent are given by (4) and (5), respectively, i.e.,

$$
\begin{aligned}
G_{m}^{o}(v, t)= & \frac{P_{r}}{\Delta v} b^{m} \operatorname{rect}\left(\frac{v-v_{0}-F^{m}(t)}{\mathrm{BW}}\right) \\
& +\left(\frac{P_{r}}{\Delta v} \sum_{j=1, j \neq m}^{K} b^{j} \operatorname{rect}\left(\frac{v-v_{0}-F^{j}(t)}{\mathrm{BW}}\right)\right) \\
& \times \operatorname{rect}\left(\frac{v-v_{0}-F^{m}(t)}{\mathrm{BW}}\right) \\
I_{m}^{o}(t)= & \Re \int_{v=0}^{\infty} G_{m}^{o}(v, t) d v \\
= & \Re b^{m} \frac{P_{r}}{\Delta v} \mathrm{BW}+\Re \frac{P_{r}}{\Delta v} \sum_{j=1, j \neq m}^{K} b^{j} \\
& \times \sum_{i=1}^{N_{m, j}}\left(\mathrm{BW}-\left|F^{m}(t)-F^{j}(t)\right|\right) \\
& \times\left(u\left(t-\tau L_{i}^{m, j}\right)-u\left(t-\tau H_{i}^{m, j}\right)\right)
\end{aligned}
$$

where $\Re=\eta e / h v_{0}$ is the responsivity of the photodetector, $\eta$ is the quantum efficiency, $e$ is the electron's charge, $h$ is Planck's constant, $N_{m, j}$ is the number of intersecting points between 
users $m$ and $j$ during the bit period, and $\tau L_{i}^{m, j}$ and $\tau H_{i}^{m, j}$ are defined as the roots of the equations [see Fig. 1(c)]

$$
\begin{aligned}
& F^{m}(t)-F^{j}(t)-\mathrm{BW}=0 \\
& F^{m}(t)-F^{j}(t)+\mathrm{BW}=0 .
\end{aligned}
$$

After the integrator and sampler, the optical photocurrent is

$$
\begin{aligned}
I_{m}= & \Re \frac{1}{T} \int_{t=0}^{T} I_{m}^{o}(t) d t \\
= & \Re b^{m} \frac{P_{r}}{\Delta v} \mathrm{BW}+\Re \frac{P_{r}}{T \Delta v} \sum_{j=1, j \neq m}^{K} b^{j} \\
& \times \sum_{i=1}^{N_{m, j}}\left(\mathrm{BW}\left(\tau H_{i}^{m, j}-\tau L_{i}^{m, j}\right)\right. \\
-\int_{\tau H_{i}^{m, j}}^{m, j} & \left.\left|F^{j}(t)-F^{m}(t)\right| d t\right) .
\end{aligned}
$$

The first term is the desired data and the second term is the MAI. For a large number of interfering users, the probability density function of the MAI is usually approximated by Gaussian distribution according to the central limit theorem.

\section{DOCDMA PERFORMANCE ANALYSIS}

In this analysis, we consider the MAI effect, the PIIN, and the thermal noise. Other sources like shot noise and receiver's dark current noise are neglected. Gaussian approximation is used for the calculation of the BER.

The photocurrent can be reformulated as

$$
I_{m}=b^{m} I+\operatorname{MAI}(m)
$$

where $I=\Re\left(P_{r} / \Delta v\right) \mathrm{BW}, \operatorname{MAI}(m)=\sum_{j=1, j \neq m}^{K} b^{j} \mathrm{DAI}(m, j)$, and

$$
\begin{aligned}
\operatorname{DAI}(m, j)=\Re \frac{P_{r}}{T \Delta v} \sum_{i=1}^{N_{m, j}} & \left(\operatorname{BW}\left(\tau H_{i}^{m, j}-\tau L_{i}^{m, j}\right)\right. \\
& \left.-\int_{\tau L_{i}^{m, j}}^{\tau H_{i}^{m, j}}\left|F^{j}(t)-F^{m}(t)\right| d t\right) .
\end{aligned}
$$

Since our system is synchronized, users $m$ and $j$ will interfere at the same points in time relative to the beginning of the bit period, and the intersecting edges $\left(\tau L_{i}^{m, j}, \tau H_{i}^{m, j}\right)$ are the same whenever users $m$ and $j$ are active. This results in a constant value of $\operatorname{DAI}(m, j)$ if users $m$ and $j$ are active, otherwise $\operatorname{DAI}(m, j)$ is zero. For equiprobable data, $\operatorname{DAI}(m, j)$ is a random variable with average

$$
\mu_{\mathrm{DAI}}=\frac{1}{K^{2}-K} \sum_{m=1}^{K} \sum_{j=1, j \neq m}^{K} \operatorname{DAI}(m, j)
$$

TABLE I

TyPical PARAmeters USED IN THE CALCUlation

\begin{tabular}{|l|l|}
\hline Parameter & Value \\
\hline Bandwidth of light source & $\Delta v=3.746 \mathrm{THz}(30 \mathrm{~nm})$ \\
\hline Operating wavelength & $v_{0}=193.5 \mathrm{THz}(1550 \mathrm{~nm})$ \\
\hline Filter bandwidth & $B W=0.1249 \mathrm{THz}(0.2 \mathrm{~nm})$ \\
\hline PD quantum efficiency & $\eta=0.6$ \\
\hline Electrical equivalent bandwidth & $B=77.5 \mathrm{MHz}$ \\
\hline Data bit rate & $B R=155 \mathrm{Mbps}$ \\
\hline Receiver noise temperature & $T_{n}=300 \mathrm{~K}$ \\
\hline Receiver load resistor & $R_{l}=1000 \Omega$ \\
\hline
\end{tabular}

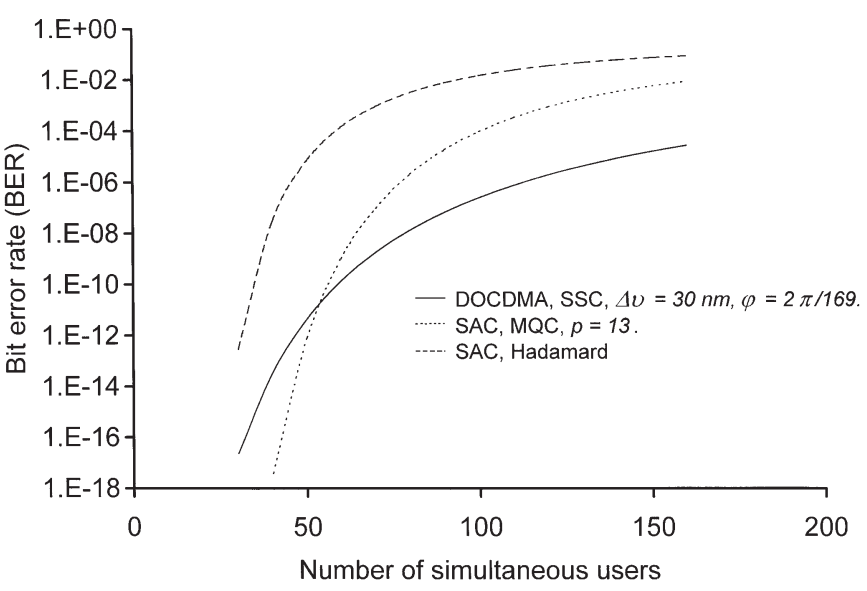

Fig. 3. Probability of error versus simultaneous active users when $P_{r}=$ $-10 \mathrm{dBm}$.

and variance

$$
\sigma_{\mathrm{DAI}}^{2}=\frac{1}{K^{2}-K} \sum_{m=1}^{K} \sum_{j=1, j \neq m}^{K}\left(\mathrm{DAI}(m, j)-\mu_{\mathrm{DAI}}\right)^{2}
$$

since we do not know which user will be active at any given time we average over all code pairs. The mean MAI can be approximated as $\mu_{\mathrm{DAI}}$, and the variance is $(K-1) \sigma_{\mathrm{DAI}}^{2}$.

Incoherent light sources mixed at the input of the photodetector will cause intensity noise in the output current (PIIN). The variance of the photocurrent due to this type of noise is

$$
\sigma_{\mathrm{PIIN}_{m}}^{2}(t)=I^{2} \tau_{c}(t) B
$$

where $\tau_{c}$ is the coherence time, and $B$ is the noise-equivalent electrical bandwidth of the receiver. The coherence time is related to the integration of the PSD as

$$
\tau_{c}(t)=\frac{\int_{v=0}^{\infty} G_{m}^{2}(v, t) d v}{\left(\int_{v=0}^{\infty} G_{m}(v, t) d v\right)^{2}}
$$

Assuming no more than one pair of users interfering at the same time, as in our proposed functional code family, the 


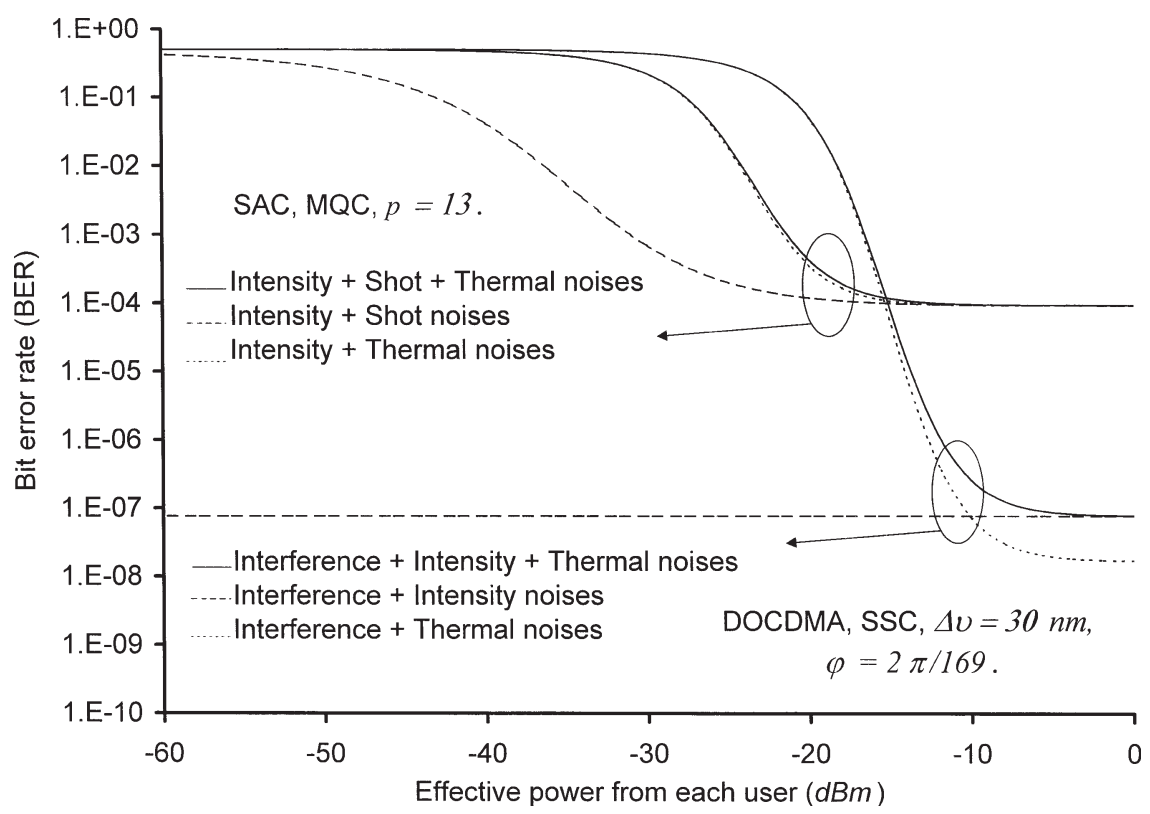

Fig. 4. Probability of error versus effective power from each user when $K=100$.

integration of the PSD squared at the time of interference and at no interference is given, respectively, as

$$
\begin{aligned}
& \left.\int_{v=0}^{\infty} G_{m}^{2}(v, t) d v\right|_{\text {Interference }} \\
& =\left(\frac{P_{r}}{\Delta v} b_{m}+\frac{P_{r}}{\Delta v} b_{j}\right)^{2}\left(\mathrm{BW}-\left|F^{m}(t)-F^{j}(t)\right|\right) \\
& \quad+\left(\frac{P_{r}}{\Delta v} b_{m}\right)^{2}\left|F^{m}(t)-F^{j}(t)\right| \\
& \int_{v=0}^{\infty} G_{m}^{2}(v, t) d v \mid \\
& =\left(\frac{P_{r}}{\Delta v} b_{m}\right)^{2} \text { BW Interference }
\end{aligned}
$$

where $j$ is the interfering user, and $i$ is the interference point.

Then, the variance of the PIIN is zero at no interference, and at the points of interference the PIIN is

$$
\begin{array}{r}
\sigma_{\mathrm{PIIN}_{m}}^{2}(t)=B \Re^{2}\left(\frac{P_{r}}{\Delta v} b_{m}+\frac{P_{r}}{\Delta v} b_{j}\right)^{2}\left(\mathrm{BW}-\mid F^{m}(t)\right. \\
\left.-F^{j}(t) \mid\right)+B \Re^{2}\left(\frac{P_{r}}{\Delta v} b_{m}\right)^{2}\left|F^{m}(t)-F^{j}(t)\right|
\end{array}
$$

averaging along the bit period and averaging over all users will get the PIIN variance equation (18), shown at the bottom of the page.

The variance of the PIIN for $k$ users can be expressed as $\sigma_{\text {PIIN }}^{2}=k \overline{\sigma_{\text {PIIN }}^{2}}$. The power of the noise sources considered can be written as

$$
\sigma_{t}^{2}=(K-1) \sigma_{\mathrm{DAI}}^{2}+\sigma_{\mathrm{PIIN}}^{2}+\frac{4 K_{b} T_{n} B}{R_{l}}
$$

where $B$ is the noise-equivalent electrical bandwidth in hertz, $K_{b}$ is Boltzmann's constant in joules per kelvin, $T_{n}$ is the absolute receiver noise temperature in kelvin, and $R_{l}$ is the receiver load resistor in ohm. The signal-to-noise ratio is

$$
\operatorname{SNR}(K)=\frac{I^{2}}{(K-1) \sigma_{\text {DAI }}^{2}+\sigma_{\mathrm{PIIN}}^{2}+\frac{4 K_{b} T_{n} B}{R_{l}}}
$$

According to the central limit theorem, we can consider that the probability density function of the variables obeys Gaussian distribution. The probability of error is thus

$$
\operatorname{BER}(K)=\frac{1}{2} \operatorname{erfc}\left(\sqrt{\frac{\operatorname{SNR}(K)}{2}}\right) .
$$

$$
\begin{array}{r}
\overline{\sigma_{\mathrm{PIIN}}^{2}}=\frac{1}{K} \sum_{m=1}^{K} \frac{1}{T} \int_{0}^{T} B \Re^{2} \sum_{j=1, j \neq m}^{K} \sum_{i=1}^{N_{m, j}}\left(\left(\frac{P_{r}}{\Delta v} b_{m}+\frac{P_{r}}{\Delta v} b_{j}\right)^{2}\left(\mathrm{BW}-\left|F^{m}(t)-F^{j}(t)\right|\right)\right. \\
\left.+\left(\frac{P_{r}}{\Delta v} b\right)^{2}\left|F^{m}(t)-F^{j}(t)\right|\right)\left(u\left(t-\tau L_{i}^{m, j}\right)-u\left(t-\tau H_{i}^{m, j}\right)\right) d t
\end{array}
$$




\section{RESULTS AND DISCUSSION}

The average variance is simulated for the sine functional code family proposed. Typical parameters used in the calculations are given in Table I. Fig. 3 shows the relation between the BER and the number of simultaneous active users when $P_{r}=$ $-10 \mathrm{dBm}$ and all three noises are considered, namely, the intensity noise, the thermal noise, and the MAI. The BER functions for two other SAC systems (one using the Hadamard code and the other using the MQC code with a prime number of 13 [23]) are also plotted in the same figure for the sake of comparison. The BER of the DOCDMA system is increasing at a slower rate than that of the other two systems, which indicates that there is a significant improvement in performance at a large number of users. Indeed, it is shown that the BER for DOCDMA is better at any number of users more than 55 . However, for less than 55 active users, the SAC system with MQC gives a BER better than that of the DOCDMA system. It should be noted that for this range of users the error rate is too small (less than $1 \times 10^{-12}$ ).

In Fig. 4, the BER is shown considering different types of noises relative to the effective power $P_{r}$ when the number of simulation users is 100 . The figure shows that the interference between users is the main source of noise that limits the system performance at large power. At low power, it is shown that thermal noise is the main factor that limits the system performance and has much effect compared to the PIIN. The same relations are shown for an SAC system with MQC code using a prime number of $p=13$. The figure also shows that the performance of the DOCDMA system is better than the SAC system with MQC code for any received power of more than $-15 \mathrm{dBm}$, and the performance is the same at smaller power level. Furthermore, the error floor of our system $\left(7 \times 10^{-8}\right)$ is much less than that of MQC $\left(1 \times 10^{-4}\right)$. This shows how our system introduces a very significant improvement for a large number of users.

\section{CONCLUSION}

We have proposed a novel low-noise dynamic optical code division multiple access (DOCDMA) communication system using functional codes. The encoder/decoder design is based on a fast tunable optical filter (TOF). The filters are controlled dynamically and move one cycle during the data bit period. Thus, the encoder and decoder are easily reconfigured to any code by changing the electrical signal set to the controller. DOCDMA with shifted sine functional (SSF) code family is analyzed taking into account multiple access interference (MAI), thermal noise, and phase-induced intensity noise (PIIN). The system shows a small bit error rate (BER) at a large number of simultaneous active users compared with other systems like SACCDMA that uses Hadamard and modified quadratic congruence (MQC) codes. The main noise affecting the performance at low effective power at the receiver is the thermal noise while at large effective power the main effect comes from the interference.

\section{ACKNOWLEDGMENT}

M. M. N. Hamarsheh would like to thank Associated Prof. M. A. Mahdi of the University Putra Malaysia, Serdang,
Malaysia, for valuable ideas and discussions and for his assistance in editing the paper.

\section{REFERENCES}

[1] J. A. Salehi, "Code division multiple-access techniques in optical fiber networks. I. Fundamental principles," IEEE Trans. Commun., vol. 37, no. 8, pp. 824-833, Aug. 1989.

[2] J. A. Salehi and C. A. Brackett, "Code division multiple-access techniques in optical fiber networks. II. Systems performance analysis," IEEE Trans. Commun., vol. 37, no. 8, pp. 834-842, Aug. 1989.

[3] E. Marom, "Optical delay line matched filters," IEEE Trans. Circuits Syst., vol. CAS-25, no. 6, pp. 360-364, Jun. 1978.

[4] K. P. Jackson, S. A. Newton, B. Moslehi, M. Tur, C. C. Cutler, J. W. Goodman, and H. J. Shaw, "Optical fiber delay line signal processing," IEEE Trans. Microw. Theory Tech., vol. MTT-33, no. 3, pp. 193-210, Mar. 1985.

[5] P. Prucnal, M. Santoro, and T. Fan, "Spread spectrum fiber-optic local area network using optical processing," J. Lightw. Technol., vol. LT-4, no. 5, pp. 547-554, May 1986.

[6] F. R. K. Chung, J. A. Salehi, and V. K. Wei, "Optical orthogonal codes: Design, analysis and applications," IEEE Trans. Inf. Theory, vol. 35, no. 3, pp. 595-604, May 1989.

[7] A. S. Holmes and R. A. Syms, "All-optical CDMA using "quasi-prime" codes," J. Lightw. Technol., vol. 10, no. 2, pp. 279-286, Feb. 1992.

[8] P. R. Prucnal, D. J. Blumenthal, and M. A. Santoro, "12.5 Gbit/s fiber optic network using all optical processing," Electron. Lett., vol. 23, no. 12, pp. 629-630, Jun. 1987.

[9] W. C. Kwonga and P. R. Prucnal, "Ultrafast all-optical code-division multiple-access (CDMA) fiber-optic networks," Comput. Netw. ISDN Syst., vol. 26, no. 6-8, pp. 1063-1086, Mar. 1994.

[10] D. D. Sampson, G. J. Pendock, and R. A. Griffin, "Photonic codedivision multiple-access communications," Fiber Integr. Opt., vol. 16, no. 2, pp. 129-157, Mar. 1997.

[11] N. Karafolas and D. Uttamchandani, "Optical fiber code division multiple access networks: A review," Opt. Fiber Technol., vol. 2, no. 2, pp. 149168, Apr. 1996.

[12] D. D. Sampson and D. A. Jackson, "Coherent optical fiber communications system using all-optical correlation processing," Opt. Lett., vol. 15, no. 10, pp. 585-587, May 1990.

[13] R. R. A. Syms, S. Makrimichalou, and A. S. Holmes, "High-speed optical signal processing potential of grating-coupled waveguide filters," Appl. Opt., vol. 30, no. 26, pp. 3762-3769, Sep. 1991.

[14] N. Wada and K.-I. Kitayama, "A $10 \mathrm{~Gb} / \mathrm{s}$ optical code division multiplexing using 8-chip optical bipolar code and coherent detection," J. Lightw. Technol., vol. 17, no. 10, pp. 1758-1765, Oct. 1999.

[15] D. D. Sampson, R. A. Griffin, and D. A. Jackson, "Photonic CDMA by coherent matched filtering using time-addressed coding in optical ladder networks," J. Lightw. Technol., vol. 12, no. 11, p. 2001, Nov. 1994.

[16] N. Karafolas and D. Uttamchandani, "Self-homodyne code division multiple access technique for fiber optic local area networks," IEEE Photon. Technol. Lett., vol. 6, no. 7, pp. 880-883, Jul. 1994.

[17] R. A. Griffin, D. D. Sampson, and D. A. Jackson, "Coherence coding for photonic code-division multiple access networks," J. Lightw. Technol., vol. 13, no. 9, pp. 1826-1837, Sep. 1995.

[18] M. Kavehrad and D. Zaccarin, "Optical code-division-multiplexed systems based on spectral encoding of noncoherent sources," J. Lightw. Technol., vol. 13, no. 3, pp. 534-545, Mar. 1995.

[19] E. D. J. Smith, R. J. Blaikie, and D. P. Taylor, "Performance enhancement of spectral-amplitude-coding optical CDMA using pulse-position modulation," IEEE Trans. Commun., vol. 46, no. 9, pp. 1176-1185, Sep. 1998.

[20] C. F. Lam, D. T. K. Tong, M. C. Wu, and E. Yablonovitch, "Spectrally encoded CDMA system using Mach-Zehnder encoder chains," in Proc. SPIE-Opt. Eng., Dallas, TX, 1998, vol. 3228, pp. 399-407.

[21] - "Experimental demonstration of bipolar optical CDMA system using a balanced transmitter and complementary spectral encoding," IEEE Photon. Technol. Lett., vol. 10, no. 10, pp. 1504-1506, Oct. 1998.

[22] X. Zhou, H. H. M. Shalaby, C. Lu, and T. Cheng, "Code for spectral amplitude coding optical CDMA systems," Electron. Lett., vol. 36, no. 8, pp. 728-729, Apr. 2000.

[23] Z. Wei, H. M. H. Shalaby, and H. Ghafouri-Shiraz, "Modified quadratic congruence codes for fiber Bragg-grating-based spectral-amplitudecoding optical CDMA systems," J. Lightw. Technol., vol. 19, no. 9, pp. 1274-1281, Sep. 2001.

[24] Z. Wei, H. Ghafouri-Shiraz, and H. M. H. Shalaby, "New code families for fiber-Bragg-grating-based spectral-amplitude-coding optical CDMA 
systems," IEEE Photon. Technol. Lett., vol. 13, no. 8, pp. 890-892, Aug. 2001.

[25] Z. Wei and H. Ghafouri-Shiraz, "Codes for spectral-amplitudecoding optical CDMA systems," J. Lightw. Technol., vol. 20, no. 8, pp. 1284-1291, Aug. 2002.

[26] - "Proposal of a novel code for spectral amplitude-coding optical CDMA systems," IEEE Photon. Technol. Lett., vol. 14, no. 3, pp. 414416, Mar. 2002.

[27] S. A. Aljunid, M. Ismail, A. R. Ramli, B. M. Ali, and M. K. Abdullah, "A new family of optical code sequences for spectral-amplitude-coding optical CDMA systems," IEEE Photon. Technol. Lett., vol. 16, no. 10, pp. 2383-2385, Oct. 2004.

[28] C.-H. Lin, J. Wu, H.-W. Tsao, and C.-L. Yang, "Spectral amplitude-coding optical CDMA system using Mach-Zehnder interferometers," J. Lightw. Technol., vol. 23, no. 4, pp. 1543-1544, Apr. 2005.

[29] C. F. Lam and E. Yablonovitch, "A fast wavelength hopped CDMA system for secure optical communications," in Proc. SPIE-Opt. Eng., Dallas, TX, 1998, vol. 3228, pp. 390-398.

[30] H. Fathallah, L. A. Rusch, and S. LaRochelle, "Passive optical fast frequency-hop CDMA communications system," J. Lightw. Technol., vol. 17, no. 3, pp. 397-405, Mar. 1999.

[31] E. Inaty, L. A. Rusch, and P. Fortier, "Multirate optical fast frequency hopping CDMA system using power control," in Proc. IEEE Global Telecommunications (GLOBECOM), San Francisco, CA, Nov. 2000, vol. 2, pp. 1221-1228.

[32] V. Baby, C. S. Bres, L. Xu, I. Glesk, and P. R. Prucnal, "Demonstration of differentiated service provisioning with 4-node $253 \mathrm{Gchip} / \mathrm{s}$ fast frequency-hopping time-spreading OCDMA," Electron. Lett., vol. 40, no. 12, pp. 755-756, Jun. 2004.

[33] I. Glesky, V. Babyz, C.-S. Bres, L. Xuz, D. Randz, and P. R. Prucnalz, "Experimental demonstration of $2.5 \mathrm{~Gb} / \mathrm{s}$ incoherent two-dimensional optical code division multiple access system," Acta Phys. Slovaca, vol. 54, no. 3, pp. 245-250, Jun. 2004

[34] D. Sadot and E. Boimovich, "Tunable optical filters for dense WDM networks," IEEE Commun. Mag., vol. 36, no. 12, pp. 50-55, Dec. 1998.

[35] I. M. I. Habbab, S. L. Woodward, and L. J. Cimini, "DBR-based tunable optical filter," IEEE Photon. Technol. Lett., vol. 2, no. 5, pp. 337-339, May 1990

[36] O. Sahlen, "Active DBR filters for $2.5 \mathrm{~Gb} / \mathrm{s}$ operation: Linewidth, crosstalk, noise, and saturation properties," J. Lightw. Technol., vol. 10, no. 11, pp. 1631-1643, Nov. 1992.

[37] I. R. Croston, A. D. Carr, N. J. Parsons, S. N. Radcliffe, and L. J. S. Ville, "Lithium niobate electro-optic tunable filter with high sidelobe suppression," Electron. Lett., vol. 29, no. 2, pp. 157-159, Jan. 1993.

[38] D. Sadot, "Ultra-fast tunable fiber-loop optical filters for dense WDM applications," in Proc. IEE Colloq. WDM Technology and Applications, London, U.K., 1997, pp. 13/1-13/5.

[39] S. Matsuo, Y. Ohiso, K. Tateno, T. Segawa, M. Kohtoku, and S. Oku, "A high-speed tunable optical filter using a semiconductor double-ring resonator," in Proc. 14th Indium Phosphide and Related Materials Conf. (IPRM), Stockholm, Sweden, 2002, pp. 467-470.

[40] T. Segawa, S. Matsuo, Y. Ohiso, T. Ishii, Y. Shibata, and H. Suzuki, "Fast tunable optical filter using cascaded Mach-Zehnder interferometers with apodized sampled gratings," IEEE Photon. Technol. Lett., vol. 17, no. 1, pp. 139-141, Jan. 2005.

[41] S. Zahedi and J. A. Salehi, "Analytical comparison of various fiber-optic CDMA receiver structures," J. Lightw. Technol., vol. 18, no. 12, pp. 17181727, Dec. 2000.

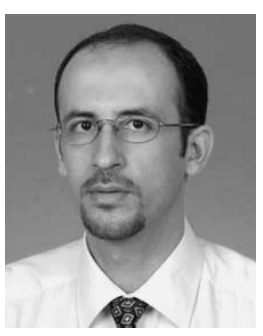

Mohammad M. N. Hamarsheh was born in Jenin, Palestine, on 1975. He received the B.Sc. degree in electrical engineering from An-najah National University, Nablus, Palestine, in 1999, the M.Sc. degree in computer and communication engineering from University Putra Malaysia, Serdang, Malaysia, in 2002, and is currently working toward the Ph.D. degree at University Putra Malaysia.

$\mathrm{He}$ is attached as a research engineer to Photronix (M) Sdn Bhd, R\&D division, Cyberjaya, Malaysia. $\mathrm{He}$ is involved in fiber Bragg grating research and development. His research interests include optical code division multiple access, systems, fiber Bragg gratings, dense wavelength division multiplexing, and polarization effect on optical fiber communication.

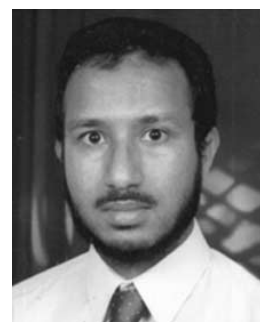

Hossam M. H. Shalaby (S'83-M'83-SM'99) was born in Giza, Egypt, on 1961. He received the B.S. and M.S. degrees from the University of Alexandria, Egypt, in 1983 and 1986, respectively, and the Ph.D. degree from the University of Maryland, College Park, MD, in 1991, all in electrical engineering.

In 1991, he was an Assistant Professor at the Department of Electrical Engineering, University of Alexandria. He was promoted to the position of Associate Professor in 1996 and then to Professor in 2001 (current position). From March to April 1996 he was a Visiting Professor at the Electrical Engineering Department, Beirut Arab University, Beirut, Lebanon. From September 1996 to January 1998, he was an Associate Professor at the Electrical and Computer Engineering Department, International Islamic University Malaysia. From February 1998 to December 1998, he was a Senior Lecturer at the School of Electrical and Electronic Engineering, Nanyang Technological University, Singapore, and from January 1999 to February 2001 was an Associate Professor. From December 2000 to 2004, he was an Adjunct Professor at the Department of Electrical and Information Engineering, Faculty of Sciences and Engineering, Laval University, Quebec City, QC, Canada. His research interests include optical communications, optical code division multiple access (CDMA), spreadspectrum communications, and information theory.

Dr. Shalaby has served as the Student Branch Counselor at the Alexandria University of IEEE Alexandria and North Delta Subsection since 2002 and served as the Chairman of the Student Activities Committee of the IEEE Alexandria Subsection from 1995 to 1996 . He has also served as a Technical Referee for ProceEdings of THE IEE, IEEE TRANSACTIONS ON COMMUNICATIONS, IEEE TRANSACTIONS ON INFORMATION THEORY, IEEE Journal on SELECTED AREAS IN COMMUnications, and the Journal of LightwaVe TeChNOLOGY. He received the SRC Fellowship from 1987 to 1991 (Systems Research Center, MD), the Shoman Prize for Young Arab Researchers in 2002 (The Abdul Hameed Shoman Foundation, Amman, Jordan), the State Award twice in 1995 and 2001 (Academy of Scientific Research and Technology, Egypt), and the University Award in 1996 (University of Alexandria). He is listed in the 14th edition of Marquis Who's Who in the World, 1997.

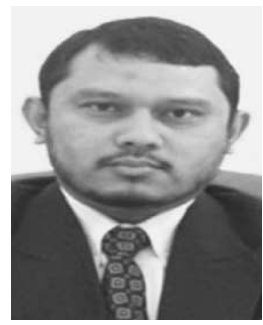

Mohamad Khazani Abdullah (S'90-M'03) was born in Terenganu, Malaysia, on 1968. He received the B.Sc. and M.Sc. degrees in electrical engineering from the University of Missouri, Rolla, in 1990 and 1993, respectively, and the Ph.D. degree from Universiti Malaya, Malaysia, in 1999

From April 1996 to August 2000, he was the Head of the Photonics Devices Unit, Research and Development Division, Telecom Malaysia. In 1999, he was an Associate Professor at the University Putra Malaysia, Serdang, Malaysia. In July 2001, he was the head of Group 6, Malaysian National Top-Down Photonics project, Ministry of Science, Technology, and Environment. He is now the head of Computer and Communication Systems Engineering Department, Faculty of Engineering, University Putra Malaysia. He currently holds eight patents for various inventions. His research interests include fiber optics devices, nonlinear optics, dense wavelength division multiplexing (DWDM) systems, and optical code division multiple access (OCDMA) systems.

Dr. Abdullah is currently the Chair of Lasers and Electro-Optics Society (LEOS), Malaysian Chapter. He was the recipient of the Malaysian National Young Scientist Award for 2002 and has received various international invention shows including Geneva and at INPEX, USA. 